\title{
Les cheminots reflétés aux miroirs de la pub'
}

Railwaymen in advertising

\section{Georges Ribeill}

\section{OpenEdition}

\section{Journals}

Édition électronique

URL : https://journals.openedition.org/rhcf/109

DOI : 10.4000/rhcf.109

Éditeur

Rails \& histoire

Édition imprimée

Date de publication : 1 décembre 2007

Pagination : 128-149

ISSN : 0996-9403

\section{Référence électronique}

Georges Ribeill, « Les cheminots reflétés aux miroirs de la pub' », Revue d'histoire des chemins de fer [En ligne], 36-37 | 2007, mis en ligne le 10 mai 2011, consulté le 22 avril 2022. URL : http:// journals.openedition.org/rhcf/109; DOI : https://doi.org/10.4000/rhcf.109 


\title{
Les cheminots reflétés aux miroirs de la pub'
}

\author{
Georges Ribeill \\ Directeur de recherche à l'École nationale des Ponts et Chaussées - \\ LATTS
}

\section{Quelques thèmes publicitaires précoces}

Dans une première recherche historique consacrée aux cheminots et achevée en 1980, nous attirions l'attention, en les reproduisant, sur deux publicités extraites de journaux syndicaux ${ }^{1}$. L'une, régulièrement parue pendant l'année 1908 dans la Tribune de la voie ferrée, l'organe officiel du très puissant Syndicat national des travailleurs des chemins de fer, affilié à la CGT, invitait les «travailleurs des chemins de fer» à adopter le «chronomètre du Travailleur [...] créé spécialement pour leur usage » par l'horloger bisontin Léon Dubois (fig. 1). Son dos était orné d’une figure allégorique entourée de la devise : "Travailleurs de tous les pays, unissez-vous pour défendre vos droits. » L'autre publicité, parue dans Le Réveil des cheminots en décembre 1911, nous révélait l'opportunisme de la maison de produits alimentaires Julien Damoy, promotrice d'un apéritif à base de quinquina, Le Cheminot... (fig. 2). Parcourons systématiquement les huit copieuses pages grand format d'un exemplaire contemporain de la Tribune de la voie ferrée : dans le numéro du 24 avril 1910, en dernière page, un quart de place est réservé à sept réclames qui se répartissent nettement selon trois thèmes. Trois annonceurs visent le lecteur engagé de la Tribune: une société d'assurances mutuelles sur la vie, la Mutuelle lyonnaise (2, rue Taitbout), émancipée semble-t-il du capitalisme... (« Pas d'actionnaires! Pas de dividendes !»); la Famille laborieuse, restaurant coopératif de Sotteville-lès-Rouen à proximité des ateliers du chemin de fer; quant au Cotillon du prolétariat, fournisseur en bannières du Syndicat national, cette maison se recommande aussi « aux camarades du Syndicat national» pour leur fournir, outre des drapeaux et insignes, des articles de fête et cotillons. Mais c'est aussi le cheminot professionnel qui est visé. Deux annonces lui proposent des chronomètres (ou régulateurs) : le bisontin Ernest Magnin («Envoi pour essai à MM. les employés de chemins de fer») et la Société des montres Nomis (Paris, 31, rue Bergère), dont le boîtier décoré en relief a été « créé exclusivement pour MM. les Employés de Chemins de fer,

1- Georges Ribeill, Le Personnel des compagnies de chemins de fer, tome 1, Des origines à nos jours, [Paris], Développement et Aménagement, 1980, p. vi et p. 531. 

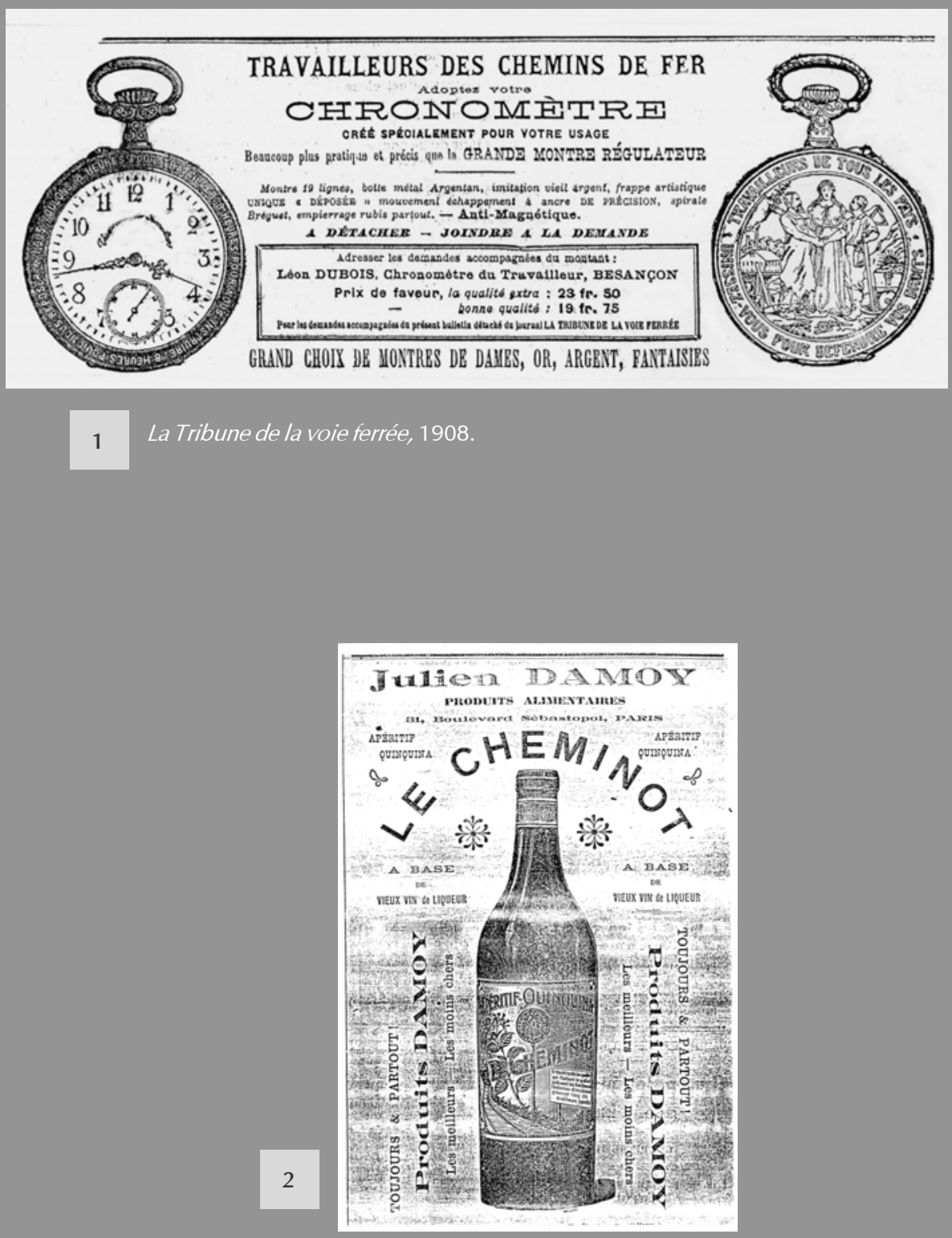

Le Réveil des cheminots, déc. 1911. 
Postes et Télégraphes ». Mais à ces « employés de chemins de fer » réputés grands buveurs de vin, Jules Reboul d'Uchaud propose, avec $10 \%$ de remise, des vins du Gard en barriques selon trois qualités (" ordinaire, supérieur ou blanc sec »), tandis que Rouquette, viticulteur à l'Ermitage de Fons, propose des « conditions spéciales aux employés de chemins de fer » qui achèteront ses pièces et demi-pièces de vin.

Si l'on consulte d'autres périodiques moins engagés, nous retrouvons encore les horlogers, par exemple dans le Bulletin officiel de l'orphelinat des chemins de fer français (juillet 1912) : toujours le bisontin Magnin et Sélecta de Morteau ; mais aussi, thème nouveau, du mobilier : « à 5 minutes des gares de l'Est et du Nord », le Palais de l'ameublement Charles Klein \& Cie (28, rue de Flandre, $\left.19^{\circ}\right)$ consent « un escompte exceptionnel de $10 \%$ aux employés de Chemins de fer sur présentation de leur carte individuelle ou un crédit de deux ans sans versement comptant» : une faveur accordée par ailleurs « aux membres de toutes les sociétés de secours mutuels des chemins de fer». Pour mieux appréhender les commerces intéressés par la corporation du rail, rien de plus parlant en fait que le Catalogue général des fournisseurs qu'édite l'Union des employés des chemins de fer PLM. Fondée en 1886, cette société au statut de coopérative de consommation ${ }^{2}$ met en avant trois commodités : dans son magasin à deux pas de la gare de Lyon (13, rue Parrot), ouvert du lundi au samedi, le cheminot adhérent trouvera des articles d'épicerie (le café Union PLM par exemple), des produits d'éclairage, blanchissage et nettoyage, mais aussi des vêtements et chaussures; il peut être livré à domicile, deux jours par semaine s'il habite dans le $12^{\mathrm{c}}$ arrondissement, deux jeudis par mois dans les autres arrondissements ; enfin un réseau très varié d'enseignes de proximité lui offre remise et/ ou escompte pour tous ses achats. Adressé aux 12500 (?) sociétaires déclarés au $1^{\text {er }}$ janvier 1906, le copieux catalogue triennal (1906-19071908) de l'Union, soit 192 pages avec deux cahiers de quatre pages en couleurs au début et à la fin, nous révèle bien certaines affinités entre fournisseurs et ménages cheminots: on y vérifie l'importance des fabriques de meubles et de literie du faubourg environnant ( $A u$ Lit sans pareil, boulevard Voltaire ; Galerie de l'Ameublement, 79, avenue LedruRollin ; Le Mobilier, 68, rue du Faubourg Saint-Antoine, etc.), mais aussi plus éloigné et déjà cité, Klein, rue de Flandre.

2- Seules les compagnies de l'Est et du PLM, hostiles à la création d'économats pour leur personnel, favorisèrent au contraire sur leur réseau des coopératives de consommation, particulièrement nombreuses au PLM. Les catalogues des économats des chemins de fer, jusqu'à ceux de la SNCF, reflètent bien l'économie ménagère des foyers cheminots, thème ici hors sujet. 
Après guerre, les mêmes objets et thèmes publicitaires se retrouvent dans le milieu coopératif cheminot. Ainsi, dans le catalogue 1924 de l'Union des agents PLM de la région marseillaise $n^{\circ} 706^{3}$, les publicités de deux magasins de bijouterie-horlogerie-orfèvrerie font appel au culte cheminot de l'exactitude horaire : « Le train que vous devez prendre... ne peut pas vous attendre! Vous ne le manquerez jamais si vous avez un Chronomètre Lux» (Millet, 12, rue d'Aix) ; « Pour ne pas manquer le train, Achetez chez Henrottin une de ses montres Élection, chef-d'œuvre de précision » (Henrottin, 133, boulevard National). À Nice, l'agent est invité à acquérir une montre de précision garantie cinq ans à la boutique $A u$ Régulateur PLM, ou encore à fréquenter le Bar Barthélémy « en face la gare PLM, ouvert jusqu'au dernier train, rendezvous de tous les Agents PLM »... Dans le catalogue triennal 1929-1930-1931 «tiré à 25000 exemplaires » (184-XII p.) de l'Union parisienne du PLM maintenant installée à deux pas de la gare de Lyon, 12 , rue Abel, nous retrouvons toujours en page de dos l'annonce de $L a$ Belle Jardinière. Par contre, se distingue bien Crozatier-Meubles, " Maison de confiance fondée en 1904 » par Maurice Eisenmann, 10, rue Crozatier, affirmant être «la Maison préférée des cheminots » : ne leur réserve-t-elle des conditions de remise (achat au comptant) ou de remboursement (achat à crédit) « toutes particulières » ? Mais toutes les autres le font aussi...

\section{Des motifs et arguments publicitaires ajustés à la cible corporative}

Ces trois grandes familles de produits ainsi tôt identifiées, chronomètres, vins, mobilier et literie, nous les retrouverons constamment dans la presse corporative jusque dans les années 19601970, qu'elle soit d'obédience syndicale ou patronale, tout particulièrement dans La Vie du rail, hebdomadaire d'actualité professionnelle et d'information sociale publié et diffusé auprès de ses agents par la $\mathrm{SNCF}^{4}$. Ces organes ont parfois sollicité leurs annonceurs en soulignant l'importance et la spécificité du public touché : que ce soit le PO illustré (30 novembre 1932) tirant à 60000 exemplaires ("Vous toucherez les

3- Décalquée de l'Union parisienne, cette association mutualiste et économique fondée le $1^{\text {er }}$ mars 1910 avait statutairement " pour but de grouper la clientèle composée des Agents et de l'adresser chez les fournisseurs décidés à faire des réductions réelles sur le prix de tous les objets nécessaires aux besoins journaliers de l'existence ».

4- Rappelons les publications des réseaux, éteintes fin 1937 avec la création de la SNCF : depuis 1929 Le Bulletin PLM et Le PO illustré (devenant Le PO-Midi illustré en 1934) ; depuis 1931, L'État, notre réseau; ces publications sont relayées par le périodique de la SNCF, Notre Métier, paru de mai 1938 jusqu’à mai 1940, reparu à la Libération (suite de la note page suivante) 
Cheminots de tous grades du Réseau d'Orléans en confiant votre publicité au PO Illustré»); ou Le Bulletin PLM «excellent véhicule de publicité sur notre réseau " (mars 1932, image rééditée par Danièle Brocheton, "Lorsque les cheminots étaient la cible des publicitaires ", La Vie du rail, 25 février 2004, p. 24 - fig. 3) ; ou encore Notre Métier (septembre 1938, réédité ibid.) soulignant son intéressant lectorat cheminot à deux titres, quantitatif ( 400000 familles deviendront vos clients ») et qualitatif ( La revue Notre Métier touche le personnel de la SNCF, clientèle au pouvoir d'achat constant »).

La fréquentation de ce corpus permet d'identifier rapidement, au-delà des trois constantes thématiques indiquées plus haut, d'autres objets et types de publicité que leur récurrence permet de qualifier de corporatifs dans la mesure où les arguments publicitaires, implicites ou explicites, se distinguant peu en fonction de leurs supports institutionnels, patronaux, syndicaux, mutualistes ou coopératifs, renvoient plutôt aux particularismes de la "société cheminote $"^{5}$. Plusieurs argumentaires, qu'ils soient d'ordre économique, fonctionnel ou symbolique, structurels ou circonstanciels, font ainsi des foyers cheminots des cibles publicitaires privilégiées, que nous recenserons ici de manière empirique. S'il est difficile bien entendu d'apprécier l'efficacité de ces publicités, elles nous révèlent plutôt comment les cheminots sont perçus par les

(suite de la note 4) à partir de septembre 1944, devenant progressivement La Vie du rail (à partir de 1952). Une très riche anthologie illustrée de publicités parues dans ces organes, dans Notre Métier principalement, a été constituée par Danièle Brocheton, documentaliste à $L a$ Vie du rail, parue le 25 février 2004, p. 19-33, et intitulée : «Lorsque les cheminots étaient la cible des publicitaires ». Nous renvoyons commodément le lecteur à quelques-unes de ces illustrations qui complètent notre propre sélection.

5- Le sujet exploratoire de cet article, plongée dans l'intimité de la culture cheminote via ses consommations domestiques, ne peut que s'inspirer avec humilité des travaux pionniers de Michel Verret sur la culture ouvrière : Michel Verret (avec la collaboration de J. Creusen), L'Espace ouvrier, Paris, Armand Colin, 1979 (rééd. L'Harmattan, 1994) ; du même (avec la collaboration de P. Nugues), Le Travail ounrier, Paris, Armand Colin, 1982 ; du même (avec la collaboration de J. Creusen), La Culture ourrière, Saint-Sébastien-sur-Loire, ACL Editions, 1988 ; enfin son recueil d'articles variés sur la classe ouvrière, Chevilles ourrières, Les Éditions de l'Atelier, 1995, 254 p. Du moins, s'agissant de la face intime de la société cheminote, dispose-t-on de recherches inspirées par son œuvre et dues à Fabienne Laurioux : «Le dimanche ouvrier », mémoire de maitrise en sociologie (dir. Jean-Paul Molinari), université de Nantes, octobre 1985, 213 p. + annexes ; "L'ouvrier dans ses meubles », thèse de doctorat en sociologie (dir. Jean-Paul Molinari), université de Nantes, 1990, 532 p. Ses ménages ouvriers étudiés sont en fait souvent des ménages cheminots, habitant le quartier du Vieux-Doulon au nord-est de Nantes. Nous recouperons ainsi à plusieurs reprises ses observations avec les nôtres. 
annonceurs et les fabricants et, donc, sollicités par les «sirènes » de la publicité par des argumentaires ajustés. Cela n'exclut pas que de nombreuses et grandes marques aient recours à leurs images et slogans publicitaires passe-partout.

\section{Crédit et facilités de paiement pour des quasi-fonctionnaires}

Du point de vue des commerçants, des fournisseurs, les cheminots, pour l'essentiel commissionnés, présentaient l'attrait de pouvoir être assimilés à des fonctionnaires, du fait de la garantie de l'emploi implicite et de leur traitement fixe ${ }^{6}$. Ainsi se comprend l'abondance des publicités pour des équipements d'un coût élevé, mais toujours assorties de facilités de paiement. Tous les marchands de salons, chambres à coucher, etc., rappellent cette faveur. Ainsi, si l'acquisition d'une chambre à coucher représente une grosse dépense, Crozatier consent d'importantes facilités de paiement, y compris par le versement de bons et titres français - obligations et actions de chemins de fer, sans doute de manière privilégiée (Notre Métier, n 8, 15 juillet 1939, fig. 4). C'est une constante qu'illustrent par exemple Lévitan (Notre Métier, 30 octobre 1950, art. cité, 2004, p. 19) ou Crozatier (Notre Métier, 25 février 1952, ibid., p. 22). Cette faveur est étendue au début des Trente Glorieuses au service de table pour le jeune ménage : «Aide aux jeunes ménages cheminots ", offre spéciale d'un ensemble de table complet de 168 pièces à $50 \%$ de sa valeur réelle, payable en sept mensualités (Notre Métier, 17 décembre 1951, ibid., p. 31), aux vêtements ADF (Notre Métier, 28 mars 1954, ibid., p. 21), jusqu'aux manteaux de fourrure Brunswick (Notre Métier, 9 juillet 1951, ibid., p. 30).

\section{Proximité ou isolement géographique}

On a déjà illustré plus haut la proximité géographique de quartier de la gare : à Crozatier, fondée en 1904 au cœur du faubourg Saint-Antoine, omniprésent entre les deux guerres dans le Bulletin PLM, les agents parisiens du PLM ; à Lévitan, « 63 boulevard Magenta, métro Gare de l'Est» (Notre Métier, 6 février 1950, fig. 5), ou aux Galeries Barbès, ceux du Nord et de l'Est ; à Bayard, 48, Chaussée-d'Antin, ceux de Saint-Lazare... Mais toujours à deux pas de la gare de Lyon,

6- La loi du 12 janvier 1895 sur la saisie-arrêt sur les salaires et petits traitements des ouvriers ou employés accordait un certain privilège en matière de saisie-arrêt relativement aux ouvriers et gens de service, ainsi qu'aux employés d'un traitement inférieur à 2000 francs. Elle plafonnait à $1 / 10^{e}$ la partie de leur salaire saisissable par les créanciers. En réalité, les compagnies accordèrent à leurs employés, quels que soient leur qualification et leur traitement, le bénéfice de dispositions sensiblement équivalentes. 


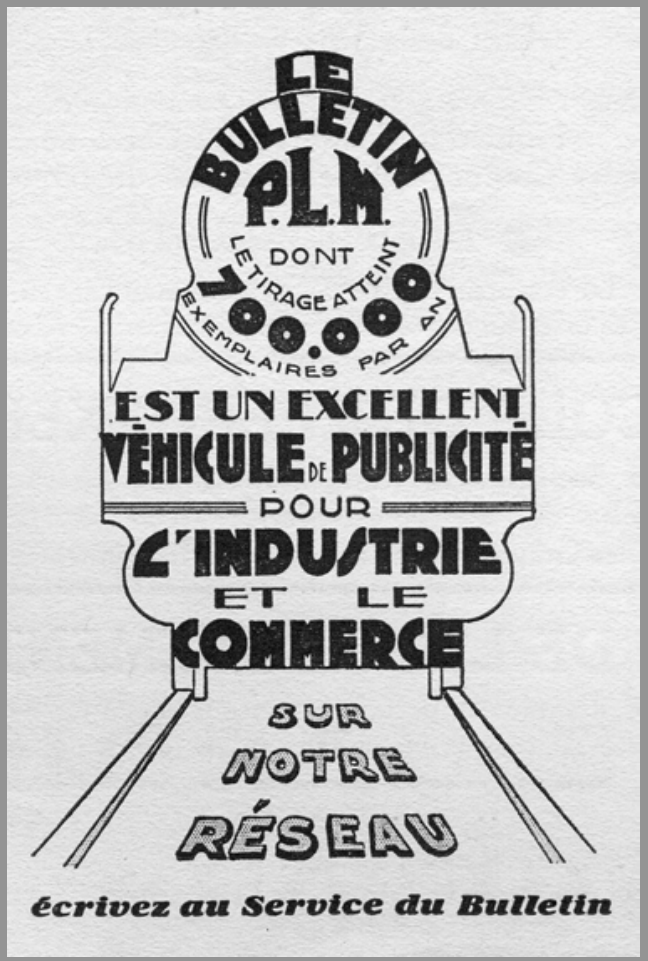

Le Bulletin PLM, mars 1932.

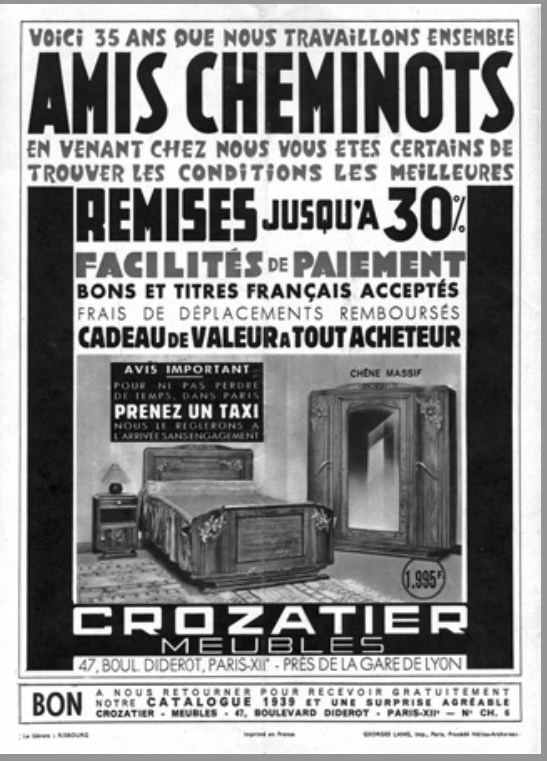

4 Notre Métier, $n^{\circ}$ 8, 15 juil. 1939.

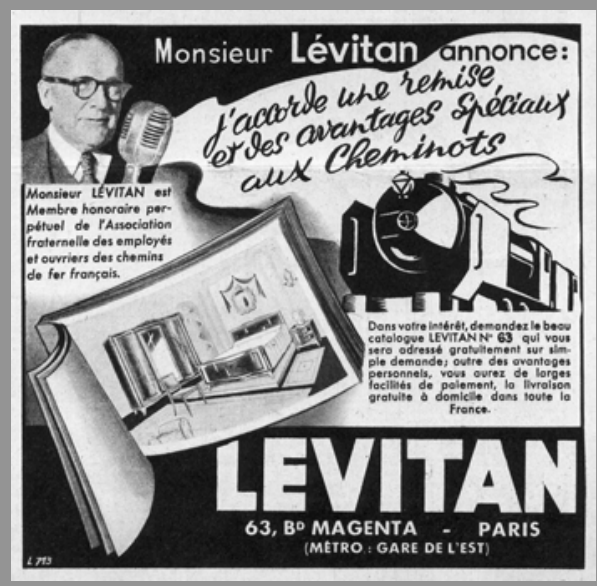

$5 \quad$ Notre Métier, n 8, 15 juil. 1939. 
12, rue Abel, siègent aussi les Pompes funèbres PLM, «service officiel des coopératives des agents de chemins de fer, Section PLM », bien qu'en 1949, date de cette citation ${ }^{7}$, il n'y ait plus d'agents PLM !

L'argument de proximité géographique peut être renforcé par un argument de proximité sociale. Ainsi J. Pacotte met en avant sa qualité d'« ancien employé des chemins de fer PLM, membre fondateur de l'Association fraternelle des employés et ouvriers des chemins de fer français et de la Société des retraites pour les deux sexes, dite la $230^{\mathrm{e}}$ » pour promouvoir son entreprise générale de convois et transports funèbres, bien située 194, rue de Charenton ${ }^{8}$. Solidaire de la corporation, dans ses nombreuses publicités, M. Lévitan rappelle qu'il est « membre honoraire perpétuel de l'Association fraternelle des employés et ouvriers des chemins de fer français », la plus importante des mutuelles cheminotes. Au demeurant, le cheminot sera mieux servi s'il se fait reconnaitre auprès de l'un des siens : l'on demandera chez Bayard « M. Gorse, ancien cheminot» (Notre métier, 19 janvier 1953 ; art. cité, 2004, p. 21), et aux Galeries Barbès, « Marcel Lafuste, ex-mécanicien de route, 31 ans de service, médaille de vermeil » (Notre Métier, 6 septembre 1953 ; ibid., 2004, p. 22).

Si le quartier cheminot prédispose à la promotion de ses services et commerces locaux, une très grande partie des agents vit en milieu rural, disséminés autour des petites gares et dans les maisons de gardebarrière. Inutile de revenir sur l'importance donc des annonceurs privilégiant la vente par correspondance dès lors qu'eux-mêmes sont géographiquement excentrés : producteurs et négociants en vin du Midi, fabricants ou distributeurs de montres franc-comtois?.

7- Publicité parue dans Visages de Seine-et-Oise, brochure éditée par la Renaissance de Seine-et-Oise vers 1949 pour le compte des élus locaux communistes.

8- Union des employés des chemins de fer PLM, Catalogue 1906-1907-1908, p. 162.

9- Il convient de rappeler que la fameuse société américaine Sears \& Roebuck est née outre-Atlantique à la fin du XIX siècle d'une alliance et d'un calcul opportuns entre un employé de la station de North Redwood de la Minneapolis and St-Louis Railroad's Cy dans le Minnesota, Richard Warren Sears, et d'un fabricant de montres à Hammond dans l'Indiana, Alvah Curtis Roebuck (cf. Boris Emmet \& John E. Jeuck, Catalogues and Counters. A History of Sears, Roebuck \& Company, Chicago, The University of Chicago Press, 1950 ; rééd. 1965). 


\section{Divers besoins professionnels fonctionnels}

C'est sous cette rubrique que nous retrouvons en premier lieu les chronomètres ou régulateurs, indispensables à de très nombreux agents : roulants, agents des gares, aiguilleurs, etc. Le culte professionnel de l'heure imprègne une certaine rigueur horaire même dans la vie privée, signifie cette publicité de la Chronométrie suisse ("Comme eux [chefs de gare avec guidon et sifflet donnant le départ d'un train], vous ferez l'heure !», Notre Métier, 27 octobre 1952, art. cité, 2004, p. 25).

Mais aussi les vins de table, aliments énergétiques bienvenus chez les cheminots soumis à des travaux très physiques, chauffeurs et cantonniers en particulier, voire les apéritifs plus ou moins alcoolisés, «boissons [réputées...] hygiéniques ». Trois réseaux, soutenant les filières viticoles et vinicoles de leur territoire, participent aux campagnes de propagande pour le vin ${ }^{10}$. Quant à St-Raphaël Quinquina, selon les annonces régulières dans Le PO illustré (mars 1933) jusque dans Notre Métierà la veille de la guerre, c'est bien un «apéritif tonique » qui est recommandé. Nulle surprise à voir l'omniprésent Ricard vanter après guerre son "vrai pastis de Marseille » auprès des contrôleurs de train de la ligne impériale, prétendument préservés de tout excès grâce à cet « apéritif de bonne humeur et de bonne santé $»^{11}$ (fig. 6).

Évidemment, le travail en plein air, les longs parcours à pied le long des voies doivent prédisposer les cheminots à acquérir des chaussures Le Trappeur (" Votre profession exige de demander beaucoup à vos chaussures ») (Notre Métier, 7 avril 1952, art. cité, 2004, p. 21) ou, bon pour les cheminots de tous les services au travail ou au repos, ce « gilet fourré canadien » du Foyer d'Or qui « se porte mieux qu'une canadienne pour la Gare, pour le Roulant, pour la Voie, pour vos Loisirs » (Notre Métier, 26 décembre 1954, ibid., 2004, p. 21).

\section{Une économie domestique autarcique}

Sous l'angle de l'économie de la consommation domestique, les compagnies ont encouragé une certaine autarcie du foyer cheminot, réduisant la dépendance de son niveau de vie des crises de cherté de la

10- Voir cette publicité pour le stand commun à la foire de Béziers du PO-Midi et de l'Association de propagande pour le vin, dans le PO-Midi illustré, juillet 1935.

11- Cette page de publicité, d'origine inconnue quant à sa date et à son support, a circulé récemment sur Internet. Elle n'a pas laissé de souvenir dans la mémoire cheminote, semble-t-il : il est vrai que l'on imagine mal la SNCF acceptant cette publicité dans $\mathrm{La}$ Vie du rail. Selon la direction de la communication de Ricard, ce serait bien toutefois une réclame authentique, une autre version ayant visé les postiers... Faute d'archives, on n'en sait toutefois pas plus... 
vie, du cours fluctuant des denrées vivrières de base : outre les économats (Nord, Ouest, PO, Midi) ou les coopératives (Est, PLM), elles lui ont vendu à des tarifs avantageux le charbon utilisé par leurs locomotives, procuré des matériaux au rebut ou de l'outillage réformé, prêté des terrains cultivables, donné des bons marchandises pour faire venir gratuitement barriques de vin ou de cidre et sacs de pommes de terre depuis la ferme natale... Parfois, l'isolement géographique du foyer cheminot, le savoir-faire pratique appris à l'atelier ont stimulé les pratiques de self help, sous ses deux formes domestiques majeures que sont le bricolage et le jardinage : loisirs typiques du dimanche cheminot, observe Fabienne Laurioux dans les cités cheminotes du Vieux-Doulon ${ }^{12}$, où « le jardin potager prime toujours sur le jardin d'agrément et la culture de légumes sur l'entretien des fleurs $»^{13}$. Ainsi, toutes les publications déjà citées des compagnies puis de la SNCF incluent régulièrement des rubriques dévolues à ces deux activités. Les pages consacrées au jardinage accueillent ainsi naturellement par exemple des publicités pour les graines du Coq Hardi ou Vilmorin (PO-Midi illustré, janvier 1934).

$\mathrm{Si}$ « la culture ouvrière a le goût de l'entretien, car cette même classe qui aime le neuf, aime aussi à garder l'usé $»^{14}$, cela semble encore plus vrai de la culture cheminote, où excelle l'art de recycler les traverses et les wagons réformés, fournissant ainsi de solides et inusables planches et abris aux cheminots de la cité du Vieux-Doulon aménageant leurs caves et cabanes de jardin ${ }^{15}$. Les rubriques de bricolage s'inspirent souvent de cet art de la récupération et du recyclage que cultive un autre magazine très populaire, le fameux Système D, journal hebdomadaire illustré du débrouillard, créé en juin 1924. Doit-on être alors surpris de voir glissée au dos de la page de couverture du Guide de la santé du cheminot, diffusé massivement par la SNCF après guerre ${ }^{16}$, une page de publicité pour l'acquisition de plans tout préparés? «Le bricolage peut être une source de bien-être pour votre foyer. Vous avez le goût au travail. Vous avez des doigts habiles. Vous avez envie de bien faire. Que vous manque-t-il pour réaliser? Des idées et des plans tout préparés que Notre Métier est précisément à même de vous fournir. » Soit, pour le foyer, égouttoir à vaisselle, fer à souder électrique, table et tabouret pliants, etc. ; pour « bébé », parc, siège portatif, fauteuil ; pour les enfants, des

12- Fabienne Laurioux, «Le dimanche ouvrier», mémoire cité, p. 56, p. 60.

13- Fabienne Laurioux, «L'ouvrier dans ses meubles », thèse citée, p. 355.

14- Michel Verret, L'Espace ourrier, op. cit., p. 139.

15- Fabienne Laurioux, "L'ouvrier dans ses meubles », thèse citée, p. 352.

16- Dr Pierre Delore, Le Guide de la santé du cheminot, Publications sociales SNCF, 1949. 
jouets, camion, chambre à coucher de poupée ou... une maquette de locomotive ! Plus subtilement, aux cheminots exposés aux intempéries, il est proposé le produit Idéalo " pour imperméabiliser soi-même et facilement tous tissus » (Notre Métier, 23 mars 1953, art. cité, 2004, p. 26).

Font pendant, à l'adresse des femmes d'agents, les rubriques de recettes de cuisine économiques ${ }^{17}$, travaux ménagers, voire ouvrages de dames. On relève toutefois, récurrentes, les publicités pour les machines à coudre, trahissant cette activité qu'exercent très fréquemment à domicile les femmes à qui la forte mobilité géographique - promotion oblige - de leur mari (agents des gares, agents des trains, mécaniciens et chauffeurs) interdit un emploi salarié durable ${ }^{18}$. La marque Excelsior nous rappelle que ses machines à coudre se trouvent autant à son magasin du boulevard Sébastopol que - gage de sérieux ? - dans les magasins de l'économat du réseau de l'État (L'Etat, notre réseau, novembre 1933). Singer propose une " offre spéciale aux familles de cheminots » pour l'achat à crédit de sa machine 15 B. 88, soldé en 12 mensualités (Notre Métier, 6 octobre 1952, art. cité, 2004, p. 31).

\section{Des aides à la promotion sociale interne}

L'univers social des chemins de fer est caractérisé par l'importance de la nécessaire formation professionnelle, acquise sur le tas à partir des grades de début non qualifiés (homme d'équipe, lampiste...), et, par voie de conséquence, par les marches de la promotion sociale possible qui s'ouvrent à ses agents, moyennant examens, ou concours internes. L'École spéciale des travaux publics (dite parfois École Eyrolles du nom de son fondateur), où une section des chemins de fer accueille chaque année des agents prometteurs sélectionnés par les réseaux, est la plus réputée des écoles proposant un enseignement par correspondance (« l'école chez soi ») aux agents se préparant aux concours internes des réseaux. De pleines pages de publicité rappellent cette opportunité (PO illustré, janvier 1934). Par ailleurs, dans le même sens, les réseaux ont créé des bibliothèques pour aider leurs agents à développer leur culture générale : «Des livres à votre portée. Votre Réseau met à votre disposition 44 bibliothèques. Utilisez-les ", rappelle Le PO illustré ( $\mathrm{n}^{\circ} 21$, mai 1932).

17- Sans doute pourra-t-elle compter sur les secours de son mari pour mettre en œuvre l'appareil complexe YAC breveté s.g.d.g. qui met « la fabrication familiale du yaourt à la portée de tous » (PO-Midi illustré, janvier 1934, novembre 1936).

18- Présence des machines à coudre soulignée dans les inventaires mobiliers établis par F. Laurioux, «L'ouvrier dans ses meubles », thèse citée, p. 381. 
Après guerre, la mode est aux méthodes nouvelles de «développement personnel » venues d'Amérique : l'Institut Pelman propose sa méthode par correspondance au cheminot non résigné qui, plutôt que de se laisser " aiguiller sur une voie de garage », veut « arriver vite et loin »: " Vous vous imposerez à vos subordonnés, gagnerez l'estime de vos chefs, avancerez en grade, apporterez l'aisance à votre foyer » (Notre Métier, 19 janvier 1953, art. cité, 2004, p. 26).

Par contre, observe Fabienne Laurioux chez un cheminot du Vieux-Doulon, cours et livres du cheminot autodidacte pourront être conservés dans le classeur métallique et le bureau de bois qui, récupérés à l'occasion d'une réforme du mobilier de l'administration cheminote, se retrouvent côte à côte dans la chambre d'enfant où ils symbolisent et concentrent «le temps des études » si sensible dans cet univers privilégié de promotion sociale inter-générationnelle qu'est la $\mathrm{SNCF}^{19}$.

\section{De la chambre à coucher au pavillon : les signes ostensi- bles de l'ascension sociale}

La persistance et la haute fréquence des publicités de marques variées proposant aux cheminots des salles à manger et/ou des chambres à coucher cossues, en imitation de styles anciens divers, doivent sans doute révéler une part bien pressentie du " rêve cheminot $»^{20}$, même les beaux meubles neufs d'apparat peuvent côtoyer les vieux meubles éclectiques hérités ou bricolés ${ }^{21}$ : l'acquisition de ce mobilier petit-bourgeois complet et assorti ne marque-t-elle une étape de cette ascension sociale, avant l'acquisition ultime du pavillon avec jardin ? Sans doute, ces rêves de mobilier et de propriété ne sont-ils pas spécifiques aux

19- Ibid., p. 191 ; croquis, p. 200.

20- Rêve "petit-bourgeois » du cheminot bien symbolisé par un dessin satirique de Tetsu, illustrant l'ouvrage de Charles Burgaux, Les Confidences d'Arsène. Souvenirs d'un chef de gare, Paris, Les Productions de Paris, 1959, p. 38. Ce qui n'exclut pas une adaptation aux symboles de la modernité, tel Ségalot proposant une «salle à manger frêne et acajou, en bois de placage » caractéristique de la « modernité » du mobilier des années 1960 (La Vie du Rail, 10 janvier 1960).

21- «Le décor ouvrier emprunte largement au code de visibilité du goût noble : motifs textiles, emblèmes de vaissellerie, meubles d'apparat, géométries du jardin-parc... Bien des choses, non l'essentiel pourtant, l'exclusivité du code. Ici, c'est l'inverse : le mélange des codes, l'exception continuelle à la règle. Pour tous les goûts vraiment : code de marque noble ; code de confort bourgeois ; code de méticulosité petite bourgeoise [...]. Et puis tout simplement le hors code : choses d'aucun prix, d'aucun style, associées sans autre principe de convenance que d'aisance et de plaisance » (M. Verret, La Culture ouvrière, op. cit., 1996, p. 50.) 
cheminots, partagés, comme le rappelle Fabienne Laurioux, par la classe ouvrière. Ainsi, du mobilier tardivement acquis ${ }^{22}$ peut-elle faire le « marqueur » de cette progression sociale ${ }^{23}$. Toutefois, dans son inventaire du mobilier que possèdent les six foyers cheminots étudiés du VieuxDoulon, nous ne retrouvons pas l'une de ces chambres à coucher typiques issues des magasins Lévitan, Barbès, Crozatier ou Bayard. Mais, selon nous, cette population cheminote qu'elle observe, composée plutôt de locataires que de propriétaires pavillonnaires, n'est pas « stabilisée », contrairement à ce qu'elle affirme ${ }^{24}$ : en effet, cinq de ces cheminots louent un appartement en HLM et un seul possède un pavillon.

Mais, comme le soulignent deux autres sociologues, dans cette appropriation mobilière et/ou immobilière ouvrière, il y a place pour un espace d'affirmation de l'autonomie ouvrière, pour la signature de la qualification manuelle professionnelle qui peut s'affirmer de l'autoconstruction à l'auto-entretien, via l'auto-réparation ${ }^{25}$, peut-être plus encore chez les cheminots que chez d'autres. Plusieurs publicités accréditent bien cette interprétation : telle cette publicité pour la peinture Le Lido (L'État, notre réseau, décembre 1932, fig. 7), explicite sur le lien entre l'agent gradé à casquette et son modeste pavillon ; ou pour ces tuyaux de fonte Métallit ("Cheminots qui construisez votre maison... »,

22- « La conquête mobilière, encore récente pour les familles populaires, ne fait pas de la classe ouvrière l'éventuel client naif et consommateur sans limites, des nouveautés mobilières. Elle demeure celle qui se meuble le plus tard, ou du moins qui acquiert ses meubles plus tard que les autres classes sociales», F. Laurioux, "L'ouvrier dans ses meubles ", thèse citée, p. 521-522.

23- « Parler du meuble dans la classe ouvrière revient donc à replacer l'histoire mobilière de cette classe dans un temps historique, où elle passe d'une identité de classe déracinée et migrante, à un temps de conquête. Conquête de l'espace et du temps privés », F. Laurioux, ibid., p. 512.

24- Ibid., p. 511.

25- Selon Guy Groux et Catherine Lévy (La Possession ouvrière. Du taudis à la propriété, Les Éditions de l'atelier, 1993, p. 184-185), si cette accession ouvrière à la propriété, cette constitution d'un patrimoine ouvrier signifient « enracinement et perpétuation au-delà de soi »- un peu d'embourgeoisement en somme -, elles confortent aussi «l'autonomie acquise par l'espace à soi » : c'est-à-dire dans un premier temps «ce qui brave les interdits fixés par le statut locatif. Elle se révèle dans l'univers du faire et de l'utile, dans l'établi du bricoleur qui matérialise souvent les usages sociaux et quotidiens des ouvriers. Aménagements des combles, réagencements des lieux, équipements "faits main", jardinage, élevage, culture du potager, reflètent la maitrise de l'espace ». Dans un second temps, cette autonomie permet de retrouver de manière estompée « le geste du métier, le savoir du compagnon, effacés par les techniques de l'industrie ». 
Notre Métier, 5 janvier 1939, art. cité, p. 22) ; ou pour la fibre de verre Isover déjà utilisée pour les wagons et qui assurera l'isolation thermique du logement (Notre Métier, 21 novembre 1954, ibid., p. 28, fig. 8).

\section{Le détournement des références et usages professionnels}

Ce dernier ressort publicitaire attire l'attention du lecteur par l'esprit, si ce n'est l'humour, qui inspire images et arguments, détonnant avec le sérieux que partagent plutôt les autres publicités. L'accroche allusive, par clin d'œil, à la corporation et à ses coutumes professionnelles ou langagières, par glissement/confusion entre références professionnelles et domestiques, vise la séduction par la connivence.

Ainsi le graphiste qui conçoit la page de publicité pour Crozatier a réussi à modeler typographiquement son slogan ("Cheminots, venez nous voir, c'est le moment!») en forme de locomotive à vapeur de type 230 ou Ten-Wheel (Notre Métier, 15 mai 1939, art. cité, 2004, p. 20). Jeux d'images, mais aussi jeux de mots, spécialité de Job : « Assez roulé pour aujourd'hui! - Non! Encore une cigarette dans du Job » (Notre Métier, 15 juillet 1939, ibid., 2004, p. 25, fig. 9), ou « Roulez vite... roulez doucement... mais roulez vos cigarettes dans du... Job, la marque centenaire » (Notre Métier, 15 mai 1940).

Contribution à la reproduction de la " grande famille PLM», les bleus de chez Franck font rêver le fiston de devenir « chauffeur comme papa » (Bulletin PLM, $n^{\circ}$ 50, mars 1937, fig. 10). Alors qu’à l'approche de Noël la marque LR lui propose «Le Rapide LR, le train marchant le plus vite dans le monde des jouets » (L'État, notre réseau, novembre 1933).

Et pourquoi ne pas adopter chez soi cette marque de charbon qui en fait au dépôt auprès des mécaniciens la réputation : "Cheminots ! Pour vos locomotives, La Briquette Grand'Combe. Pour vos cuisinières, Le Boulet Le Croissant. Exigez-les dans tous les dépôts » (Notre Métier, n 6, 15 mars 1939, « Supplément Sud-Est ») ; «Fournisseur de la SNCF » en pendules, Grillet se positionne aussi comme « l'horloger des cheminots » dans Notre Métier (1939) ; à la veille des congés estivaux, la notoriété de ses locomotives électriques autorise Als-Thom à inviter les épouses d'agents à s'équiper d'une panoplie d'accessoires électriques variés (chauffe-plat, sèche-cheveux, grille-pain, fer à repasser et cafetière) qui contribueront à rendre plus agréables leurs vacances : « Emportez en vacances votre confort » (Bulletin PLM, $\mathrm{n}^{\circ} 28$, juillet 1933, fig. 11). 


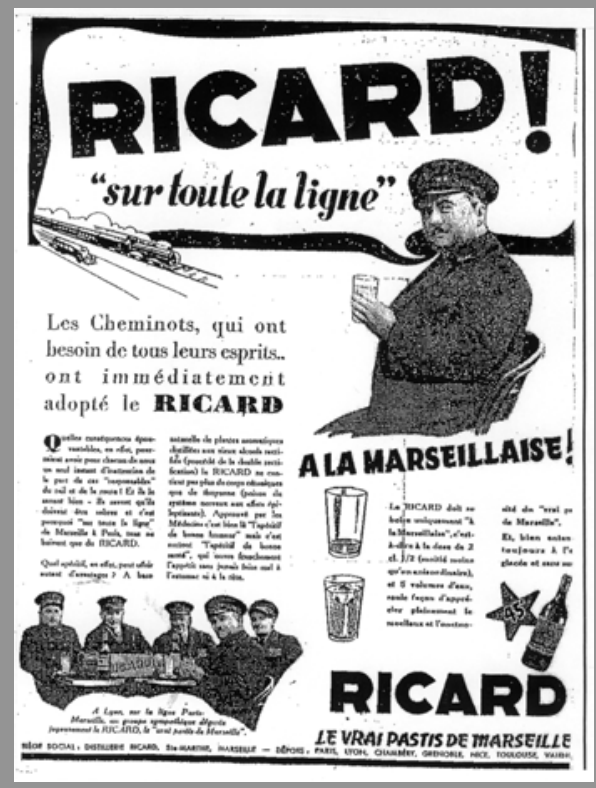

Les Cheminots, qui ont besoin de tous leurs esprits.. ont immédiatement adopté le RICART

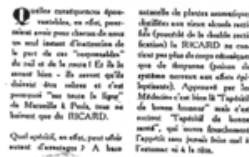

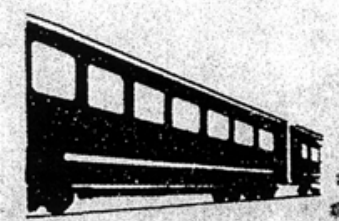

Tous les wagons sont isolés avec de la FIBRE DE VERRE :

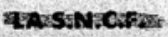
ROUREATTSONFIANCE:

Adohtez.lachezrons. now améliorestoke bien èke

Avant I'hiver, Isolez vos combles. Vous réaliserez oinsi une importante b́conomio de chauffage et votre confort sera augmenté.

Parmi les différents prodults à votre disposition, choisissex le FEUTRE NU, enroule popier que vous étendrez sur le plancher de votre grenier ou entre les solives. Le feutre de FIBRE DE VERRE se coupe sans difficulté et ne dégage aucune poussière novice.

\section{S O V E R THERMOVER
S'GOBAIN
BOUSSOIS}

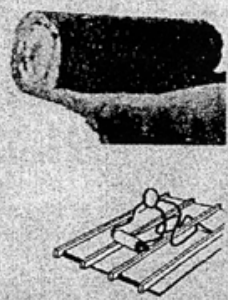

FiBRIVER Agent General pour lo france
20. Aronue Montoigne. PARIS 8

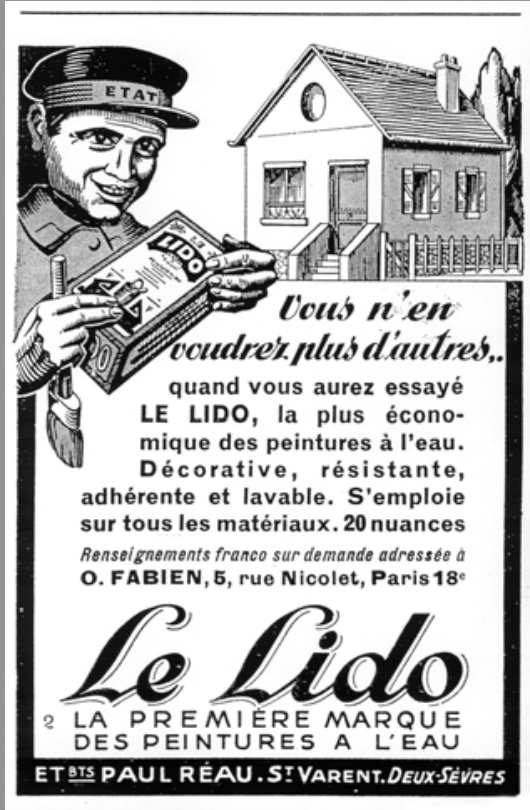

8

Notre Métier, 21 nov. 1954. 
Notre Métier, 15 mai 1940.
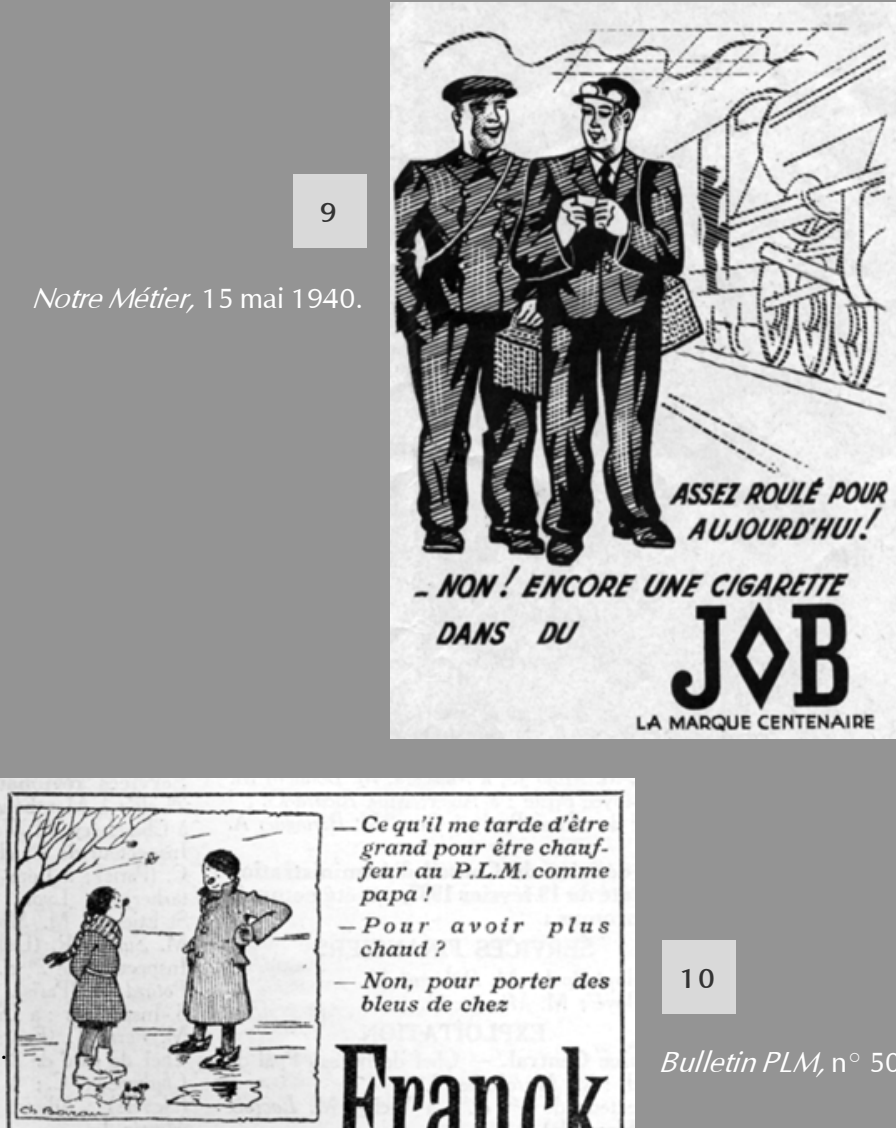

13, Rue Meslay, PARIS-3e Succursale à Nimes Passage Guérin

- Ce qu'il me tarde d'ètre grand pour être chauffeur au P.L.M. comme papa!

- Pour avoir plus chaud?

Non, pour porter des bleus de chez

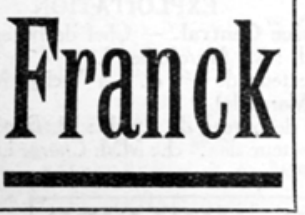

Bulletin PLM, $\mathrm{n}^{\circ}$ 50, mars 1937.
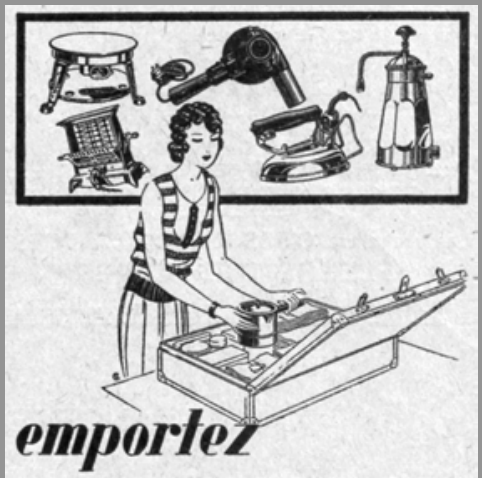
ell Virvilices volre confort ALSTHOM 
La culture cheminote et les références ferroviaires peuvent être prises à contre-pied, pour proposer à l'agent de transgresser sa modeste condition cheminote. Par exemple, par l'accès à l'automobile : «Si vous aviez une auto... CHEMINOT, ce rêve peut devenir une réalité... en participant à la Loterie au profit des œuvres sociales du PO » (PO illustré, janvier 1930); ou par l'oubli intermittent de ses vêtements de travail : Esders et La Belle Jardinière proposent concurremment au cheminot au repos de délaisser son uniforme pour un élégant costume : «Le dimanche, avec Esders, vous serez un tout autre homme » (Notre Métier, 26 février 1951, art. cité, 2004, p. 25, fig. 12) ; "Avec votre BJ, retrouvez votre personnalité » (Notre Métier, 31 mars 1952, ibid., p. 25, fig. 13).

\section{La fin de la "société cheminote"}

Nous avons développé depuis longtemps la thèse de la fin de la société cheminote, lente érosion des traditionnelles filières de recrutement (exode rural, hérédité professionnelle), amorcée durant les années 1970. Aux cheminots, formés par l'entreprise en début de carrière aux valeurs historiques de la culture ferroviaire ${ }^{26}$, ont succédé des « agents SNCF », d'origine plus urbaine, plus longtemps scolarisés et socialisés avant leur embauche à la SNCF. Ce n'est plus la vocation cheminote, l'appel du service public ferroviaire, la séduction d'un statut protecteur ou l'attrait d'une entreprise perçue à la pointe du progrès technique qui motivent l'entrée à la SNCF. Dans les bagages de ces nouvelles recrues, d'autres valeurs et attentes plus individualistes et carriéristes leur font percevoir au contraire le monde cheminot comme un monde coincé par la discipline et l'autorité, paralysé par des règlements sociaux archaïques où l'ancienneté fonde une légitimité de l'autorité douteuse et s'identifie anormalement au principal moteur de l'avancement professionnel, où le double et pesant patronage précité, encadrement professionnel et social contrôlé, n'est plus supportable. Ainsi la distanciation critique par rapport à la coutume cheminote véhiculée par les anciens, le refus de l'abandon de sa personnalité dans la vaste société cheminote anonyme ont induit le rejet des valeurs et symboles de la " grande famille du rail » et une césure franche et délibérée entre vie de travail et vie privée.

26- Technicité, exactitude horaire et respect des règles de sécurité, abnégation de soi, disponibilité et dévouement au service 24 heures sur 24, solidarité et coopération au sein des petites équipes, promotion assurée à l'ancienneté et/ou améliorée au mérite personnel, corporatisme professionnel et social introverti mais aussi double tutelle sociale patronale et syndicale, attachement aux nombreux privilèges corporatifs. 

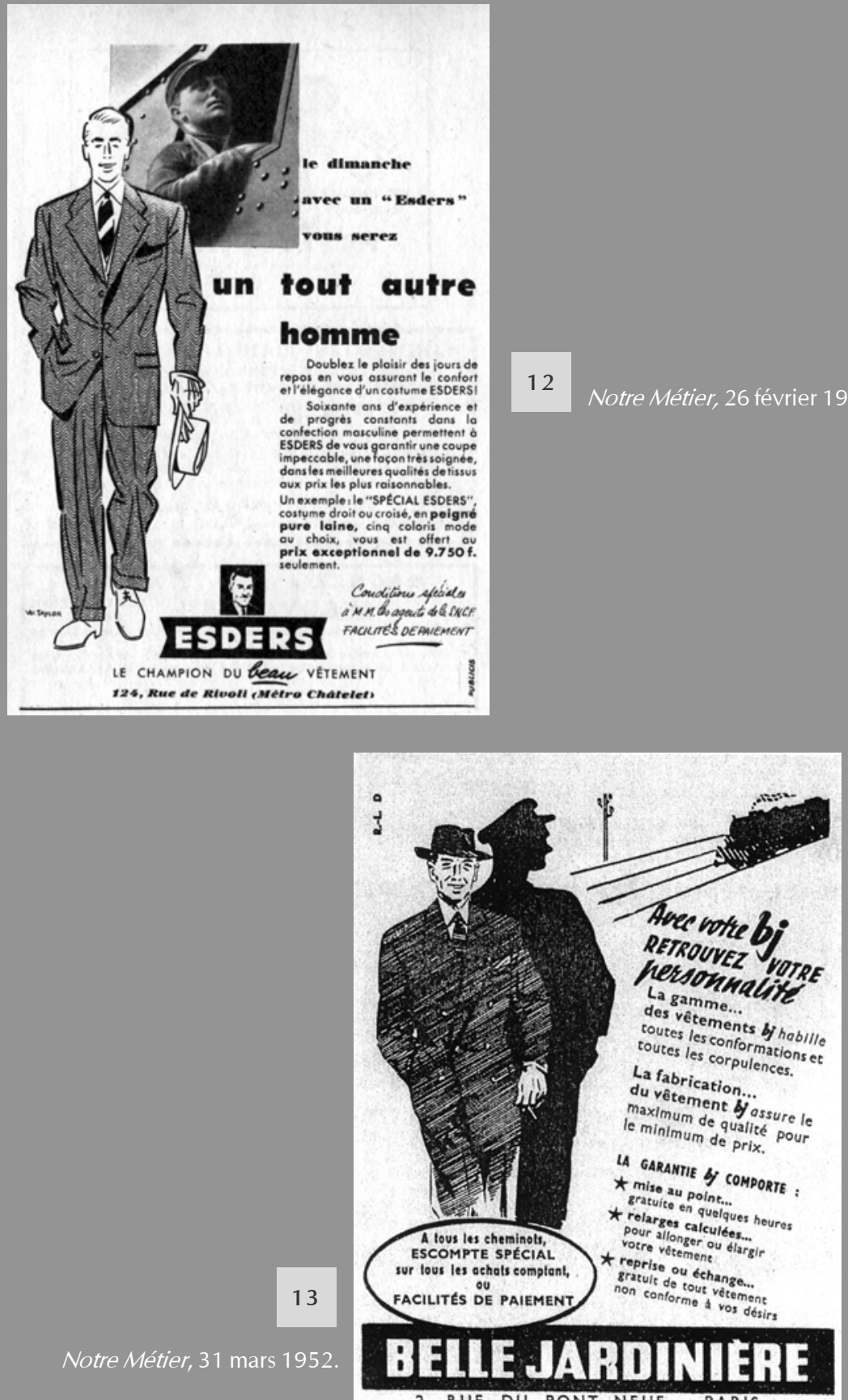

2. RUE DU PONT-NEUF - PARIS

Succursoles : Angers - Bordeaux - Lyon - Marseille - Mancy - Mantes - Saintes 
L'évolution générale de la société française durant la fin du siècle dernier a contribué elle aussi à remodeler le «ménage cheminot». En 1962, seulement dans un quart des couples dont l'un est cheminot, le conjoint (la femme essentiellement) est actif hors SNCF, et il y a bien sûr quelques couples cheminots. Mais cette donnée signifie qu'alors $70 \%$ des épouses de cheminots sont de simples "ménagères au foyer ", tributaires si ce n'est captives en somme de la « société cheminote ». Mais depuis les années 1970, «l'émancipation» de l'épouse hors de cet espace social et culturel confiné par son activité professionnelle hors SNCF a explosé : de $36 \%$ en 1973, le taux de « conjoints salariés hors SNCF » a atteint $52 \%$ en 1983, $71 \%$ en $1998,86 \%$ en $2005^{27}$ ! Ce n'est pas là l'un des moindres moteurs de sa dislocation que cette confrontation quotidienne et privée de la « société cheminote » dans le couple d'actifs avec la « société industrielle de consommation » et ses autres valeurs.

La fin de la traction à vapeur à la SNCF (vers 1970) a contribué à la banalisation des métiers du rail, tout en induisant une forte nostalgie du temps des « gueules noires » de la traction et de leurs machines. C'est bien à cette époque d'ailleurs qu'apparaissent, très nombreuses, les publicités d'objets de toutes sortes décorés de locomotives à vapeur: plaques de cheminée (La Vie du rail, 2 janvier 1966), services ou sets de table (La Vie du rail, 12 juillet 1970), planche à fromage, verres à liqueur, chopes de bière ou pots à épices (La Vie du rail, 22 novembre 1970), le comble du kitsch ferroviaire étant sans doute atteint avec cette locomotive Buddicom au $1 / 25^{\circ}$ recélant... un poste à transistors ! ( $L a$ Vie du rail, 13 septembre 1970, fig. 14). Les dernières enquêtes du Comité central d'entreprise (CCE) de la SNCF sur les attentes des jeunes agents et auprès des agents retraités en matière de loisirs pratiqués et/ ou souhaités confirment cette distance croissante avec la culture cheminote traditionnelle que nous relevions déjà en 1993 à travers divers indices ${ }^{28}$.

Une première enquête réalisée durant le $1^{\text {ex }}$ trimestre 1996 auprès d'un échantillon représentatif de la population cheminote retraitée (960 répondants $)^{29}$ révèle le " profil moyen » de ces agents : d'un âge moyen de 67,6 ans, plus de 6 sur 10 résident dans une ville, 7 sur 10 habitent un pavillon, et plus de 8 sur 10 sont propriétaires de leur logement. Le

27- Ces taux sont établis à partir des effectifs détaillés des divers assurés sociaux au 31 décembre dont font état les rapports annuels de la Caisse de prévoyance de la SNCF.

28- "Cheminots en privé », in Maurice Lemoine ; Georges Ribeill ; Anna Malan, Les Cheminots. Que reste-t-il de la "grande famille»?, Paris, Syros, 1993, p. 179-189.

29- A. Kerbal, D. Sanchis, Enquête sur les besoins des cheminots retraités et les activités des CE et du CCE de la SNCF, Paris, Degest/CCE SNCF, janvier 1997. 


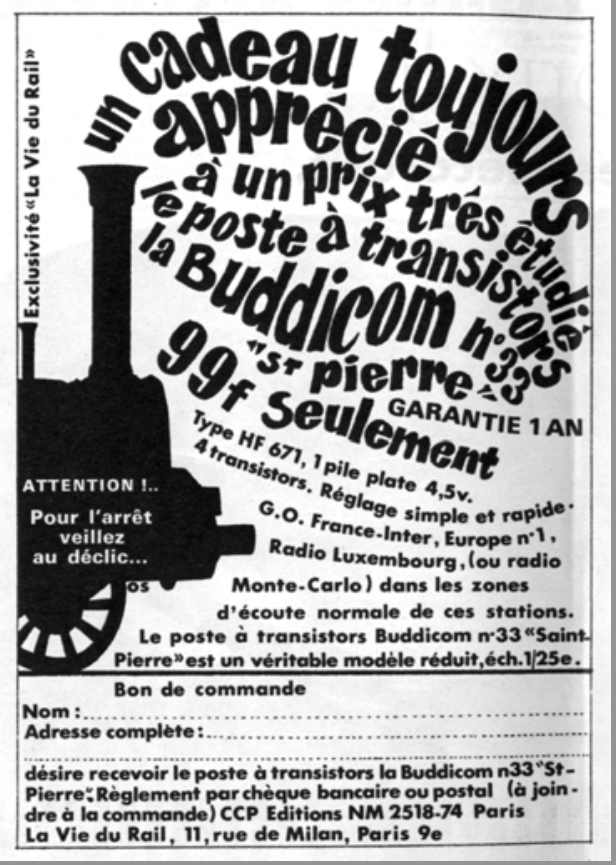

La Vie du rail, 13 septembre 1970. dre a la commande) CCP Editions NM 2518.74 Paris

La Vie du Rail, 11, rue de Milan, Paris $9 e$

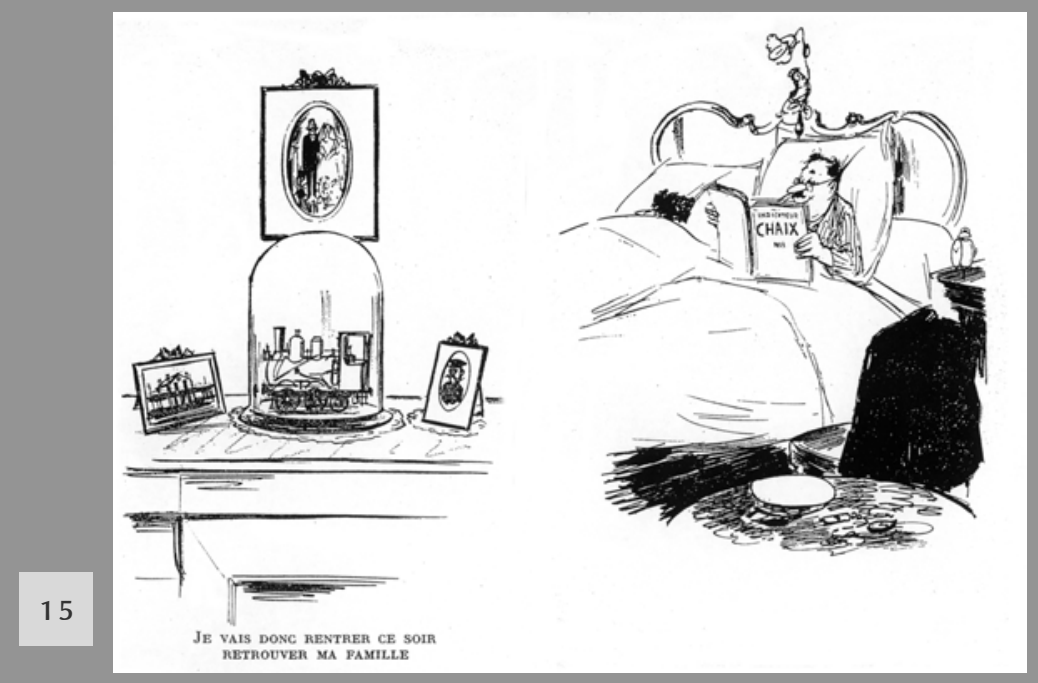

Charles Burgaux, Les Confidences d'Arsène. Souvenirs d'un chef de gare, illustré par Tetsu, Paris, Les Productions de Paris, 1959. 
modèle pavillonnaire prédomine donc, qui explique les loisirs les plus pratiqués : le jardinage puis la lecture (respectivement $67 \%$ et $49 \%$ ). En particulier, $25 \%$ adhèrent à la société du Jardin du cheminot, loin devant l'Union artistique et intellectuelle des cheminots de France (UAICF, $3 \%$ ) et l'Union sportive des cheminots de France (USCF, $2 \%$ ). Mais faut-il rappeler que la jardinage a bien évolué et qu'il est devenu moins alimentaire et plus récréatif ? Si la souche cheminote est bien prégnante dans cet échantillon $-43 \%$ ont eu des parents ou grands-parents cheminots, et seulement $37 \%$ pour les moins de 60 ans -, la méconnaissance des activités du CCE et des CE (créés au milieu des années 1980) que certains n'ont pas connus durant leur vie active contribue à alimenter le « sentiment plus ou moins exprimé (mais toujours ressenti) d'exclusion progressive de la famille cheminote » à laquelle ces retraités se réfèrent encore $^{30}$.

Une seconde enquête a été effectuée en mai-juin 2000 auprès de « jeunes agents » de moins de 30 ans (1 160 répondants) ${ }^{31}$, voire de « jeunes embauchés » (pour les trois quarts en effet, ils ont moins de cinq ans d'ancienneté à la SNCF) : $34 \%$ sont issus d'une famille cheminote (enfants ou petits-enfants) et $54 \%$ sont mariés ou vivent maritalement. S'ils sont nombreux à participer à des loisirs associatifs sportifs (23\%) ou culturels $(9 \%)$, ils ne sont toutefois plus que 2 à $4 \%$ à participer aux grandes sociétés d'agents (USCF : $4 \%$; ATC (Association touristique des cheminots) $: 3 \%$; UAICF $: 2 \%)^{32}$. Cette inadaptation de l'offre est encore plus accentuée quand il s'agit de l'offre de produits " vacances", jugée «trop orientée vers une politique de la famille», alors qu'il faudrait «diversifier un peu plus les activités afin de sortir du cadre "cheminots entre eux" ». «Il n'y a pas ce que je cherche ; il n'y a rien qui me permet de partir avec ma copine. Exemple : appart, studio, bungalow pour 2 avec des activités ", dénonce un agent. Plus largement encore, c'est bien la coupure totale avec le monde cheminot qui est recherchée ${ }^{33}$, comme l'énonce ce même agent: «Pour moi, c'est le fait de travailler une année avec des cheminots et être dans l'ambiance SNCF puis de partir en vacances avec des cheminots qui me déplait. Il faut une coupure et ne pas rester dans son environnement tout le temps ${ }^{34}$. »

30- Ibid., p. 15.

31- A. Kerbal, D. Sanchis, Enquête Jeunes cheminots (18-30 ans) : vos besoins et les activités des CE et du CCE de la SNCF, Paris, Degest/CCE SNCF, décembre 2000.

32- Ibid., p. 55.

33- Ibid., p. 17.

34- Réponse $\mathbf{n}^{\circ} 353$ à la question libre $\mathbf{n}^{\circ} 74$, ibid., p. 135. 
Si la fréquentation en 2004 des 60 centres de vacances du catalogue du CCE s'élève à 14623 enfants contre 37798 en 1986, c'est que cette évasion corporative proposée par le CCE dans «ses centres de vacances » n'est plus appropriée, même si la baisse de la fréquentation peut être expliquée en partie par la mutation profonde de la structure familiale, «le taux de divorce élevé et le développement de la garde alternée $»^{35}$.

L'apparition à l'automne $2005^{36}$ dans La Vie du rail, hebdomadaire aujourd'hui totalement émancipé de la SNCF, d'une première pleine page de publicité pour le distributeur Concorde - vente (discrète) par correspondance et en supermarchés spécialisés d'objets, vidéos, lingerie et accessoires érotiques -, proposant l'envoi gratuit de son catalogue («Le $1^{\text {er }}$ catalogue pour les plaisirs du couple. Toutes spécialités ») reflète cette mutation du cheminot qui n'est plus perçu comme voué à potasser dans son lit l'Indicateur horaire Chaix (allusion à l'un des dessins satiriques de Tetsu, fig. 15) ou à assurer la reproduction biologique de la famille cheminote... S'intéressant à l'autre extrémité de la vie de l'agent, 12 , rue Abel, près de la gare de Lyon, les Pompes funèbres PLM citées plus haut sont devenues aujourd'hui une société du Groupe Parthénos, PLM Services funéraires, dont la plaquette de présentation suggère laconiquement de nécessaires évolutions depuis sa création, fût-ce sous la bannière inchangée du PLM : « Depuis 1936, PLM, anciennement société de pompes funèbres des cheminots de la $\mathrm{SNCF}$, a développé ses structures »... Au cours d'une visite récente dans cette boutique, son gérant nous confirme bien la mort de la " grande famille » avec nostalgie : « Autrefois, nous avions une clientèle assurée et prépondérante de cheminots du quartier et de leurs familles... Aujourd'hui, nous ne traitons plus qu'avec une infime minorité d'entre eux, non significative au sein de notre clientèle. Le bon temps de la grande famille du rail qui avait suscité dans les années 1930 notre création et assuré notre développement, est bien révolu »...

35- «Vacances enfance-jeunesse : enquête d'avenir », $V u$ du Rail. Cheminot CCE, $\mathbf{n}^{\circ} 45$ (1 ${ }^{\text {er }}$ trimestre 2005), p. 4.

36- La Vie du rail (Magaqine), $\mathrm{n}^{\circ} 3018$ (28 septembre 2005), p. 35 ; page de publicité reprise à l'identique une deuxième fois dans le $\mathrm{n}^{\circ} 3021$ (19 octobre 2005), p. 29. 